\title{
P-element Somatic Inhibitor Protein Binding a Target Sequence in $d s x$ Pre-mRNA Conserved in Bombyx mori and Spodoptera litura
}

\author{
Yao Wang ${ }^{1}$, Qin Zhao ${ }^{1}$, Qiu-Xing Wan ${ }^{1}$, Kai-Xuan Wang ${ }^{1}$ and Xing-Fu Zha ${ }^{1,2,3, *}$ \\ 1 State Key Laboratory of Silkworm Genome Biology, Biological Science Research Center, Southwest \\ University, Beibei, Chongqing 400715, China; tifa1224@163.com (Y.W.); 15516767013@163.com (Q.Z.); \\ Wanqiux@outlook.com (Q.-X.W.); wkx7418@email.swu.edu.cn (K.-X.W.) \\ 3 Chongqing Engineering and Technology Research Center for Novel Silk Materials, Southwest University, \\ Chongqing 400715, China \\ * Correspondence: xfzha@swu.edu.cn; Tel.: +86-23-6825-1573; Fax: +86-23-6825-1128
}

Received: 15 April 2019; Accepted: 10 May 2019; Published: 13 May 2019

\begin{abstract}
Bombyx mori doublesex $(B m d s x)$ functions as a double-switch gene in the final step of the sex-determination cascade in the silkworm Bombyx mori. The P-element somatic inhibitor (PSI) protein in $B$. mori interacts with Bmdsx pre-mRNA in CE1 as an exonic splicing silencer to promote male-specific splicing of $B m d s x$. However, the character of the interaction between BmPSI and $B m d s x$ pre-mRNA remains unclear. Electrophoretic mobility shift assay (EMSA) results showed that the four KH_1 motifs in BmPSI are all essential for the binding, especially the former two KH_1 motifs. Three active sites (I116, L127, and IGGI) in the KH_1 motif were found to be necessary for the binding through EMSA, circular dichroism (CD) spectroscopy, and isothermal titration calorimetry (ITC). The PSI homologous protein in S. litura (SIPSI) was purified and the binding of SIPSI and CE1 was verified. Compared with BmPSI, the mutant SIPSI proteins of I116 and IGGI lost their ability to bind to CE1. In conclusion, the binding of PSI and $d s x$ pre-mRNA are generally conserved in both B. mori and S. litura. These findings provide clues for sex determination in Lepidoptera.
\end{abstract}

Keywords: Bombyx mori; Spodoptera litura; sex determination; BmPSI; KH_1 motif; electrophoretic mobility shift assay (EMSA); isothermal titration calorimetry (ITC); circular dichroism (CD) spectroscopy

\section{Introduction}

Sex determination is a fundamental process but has been found to vary among different taxa. Sex determination mechanisms in the silkworm Bombyx mori differ from those in Drosophila melanogaster, especially the upstream of $d s x$ which functions as a double-switch gene in the final step of the sex-determination cascade. In 2001, a double-switch gene, Bmdsx, was found at the final step in the sex-determination cascade of $B$. mori [1,2]. When binding to a particular sequence splicing repressor, the female-specific splice sites in $B m d s x$ pre-mRNA are inhibited, leading to the male-specific alternative splicing of Bmdsx [3,4]. A series of mutation analyses using an in vivo splicing assay system identified three distinct sequences (CE1, CE2, and CE3) in exon 4 to be exonic splicing silencers responsible for male-specific splicing of Bmdsx. The CE1 sequence was shown to bind to the nuclear protein BmPSI. Using UAA repeats to replace CE1 inhibited the binding of BmPSI to the RNA and caused female-specific splicing of Bmdsx in male cells [5]. The transgenic knockout strain of BmPSI had a defective gonad in males [6]. Then, the interaction between BmPSI and CE1 was shown to be essential for sex determination of $B$. mori by silencing the female-specific alternative splicing of 
$B m d s x$. Hence, research on binding characteristics will contribute to the discovery of the mechanisms underlying these alternative splicing processes during sex determination in many species.

BmPSI has four KH_1 motifs. The hnRNP K-homology (KH) motif has been shown to participate in sequence-specific recognition of target RNA in many species [7,8]. For example, the human PSI homolog KSRP contains four KH domains and participates in the alternative splicing of neuron-specific c-src pre-mRNA $[9,10]$. Furthermore, Drosophila PSI regulates the splicing of P-element transposase pre-mRNA by binding a pseudo-splice site upstream of the authentic splice site using four $\mathrm{KH}$ motifs [11-13]. Though there have been a few previous studies regarding these special proteins with multiple $\mathrm{KH}$ motifs, there is still little known about the detail contribution of the KH_1 motif and the key amino acids, except for the Ile-Gly-X2-Gly-X2-Ile structure, which is conservative in the KH_1 domain $[14,15]$. BmPSI contains four KH_1 motifs for binding RNA and there is a certain interaction between BmPSI and CE1. Thus, it is a good model protein in which to explore the contribution of the $\mathrm{KH}$ motif to recognize and bind RNA.

\section{Results}

2.1. Four KH_1 Motifs Play Important Roles in the Interaction Between BmPSI and CE1, Especially the KH_1-1 Motif and KH_1-2 Motif

According to the sequence of BmPSI, the tertiary structure of BmPSI was predicted to contain four KH_1 motifs and two other motifs of unknown function. The KH_1 motif was found to be an RNA binding motif in vitro. Therefore, we speculated that these four $\mathrm{KH} \_1$ motifs play important roles in the interaction between BmPSI and CE1. We purified maltose binding protein (MBP), wild-type BmPSI, and four truncate BmPSI proteins that respectively truncated $\mathrm{KH} \_1-1, \mathrm{KH} \_1-2, \mathrm{KH} \_1-3$, and KH_1-4 (Figure 1a). The concentrations of these proteins were adjusted to be identical to that of a bicinchoninic acid assay (BCA), and final concentrations of the adjustment were confirmed by sodium dodecyl sulfate-polyacrylamide gel electrophoresis (SDS-PAGE) (Figure 1b). After the concentrations of these six proteins were adjusted uniformly, they were used for an electrophoretic mobility shift assay (EMSA) (Figure 1c). As shown by the EMSA, MBP cannot bind to CE1, and the four mutant proteins almost lost their ability to interact with CE1, especially the $\Delta \mathrm{KH} \_1-1$ and $\Delta \mathrm{KH} \_1-2$, compared with the wild-type BmPSI, which was used as a positive control. In order to eliminate the effect of nonspecific binding, wild-type BmPSI, MBP- $\triangle$ KH_1-3, and MBP- $\triangle$ KH_1-4 proteins were used in a cold competition EMSA experiment (Figure 1d). When the concentration of the competitive probe was aggrandized, the signal intensity of the complex decreased sharply. These results showed that the interaction between CE1 and these three proteins are specific. Hence, we deduced that these four $\mathrm{KH} \_1$ motifs participate in the interaction between BmPSI and CE1. In addition, there was absolutely no binding signal found in the lines of MBP- $\triangle \mathrm{KH} \_1-1$ and MBP- $\Delta \mathrm{KH} \_1-2$, which was in contrast to the stable binding signal in the lines of MBP- $\triangle \mathrm{KH} \_1-3$ and MBP- $\Delta \mathrm{KH} \_1-4$. This result indicates that the KH_1-1 and KH_1-2 motifs play more important roles than the other two KH_1 motifs in the binding affinity of BmPSI. 


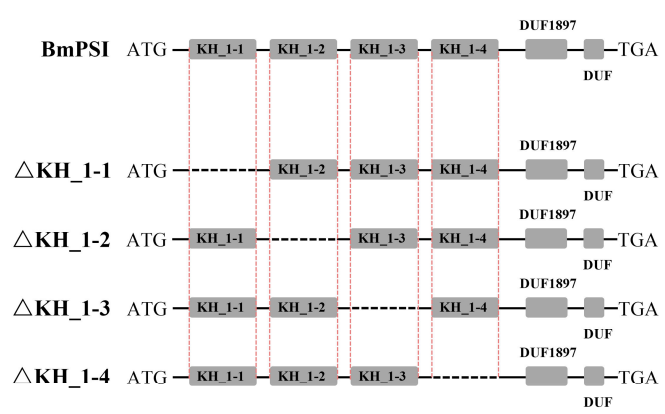

(a)

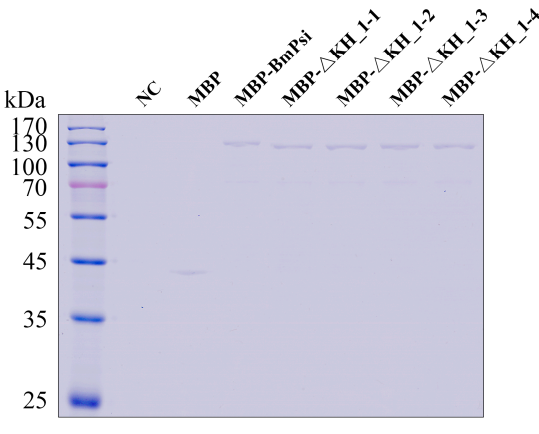

(b)

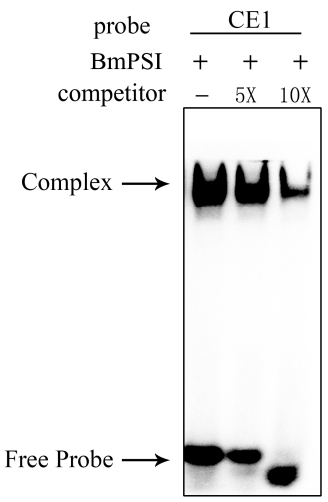

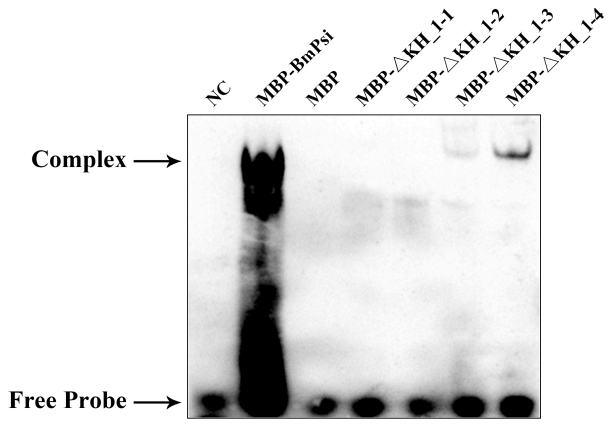

(c)

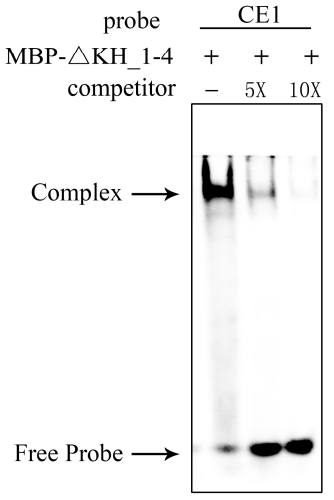

(d)

Figure 1. Structure and binding ability of BmPSI and truncation proteins: (a) Comparison among BmPSI and four truncation proteins used for the binding experiment. "DUF" means "domain of unknown function". (b) The dosage of these proteins. "NC" means "negative control". (c) Binding ability of these proteins with CE1. Buffer and maltose binding protein (MBP) were set as the negative control and wild-type BmPSI was used as the positive control. (d) Cold competition electrophoretic mobility shift assay (EMSA) experiment between CE1 and BmPSI, MBP- $\Delta K H \_1-3$, and MBP- $\Delta$ KH_1-4 proteins.

\subsection{Important Amino Acids in the KH_1-1 Motif of BmPSI Found in the Interaction between BmPSI and CE1}

We have discussed how KH_1-1 and KH_1-2 play important roles in the interaction between BmPSI and CE1. Through the comparison of the four KH_1 motifs of BmPSI, seven potential key amino acids were predicted (Figure 2a). After cloning these amino acid mutant BmPSI proteins, the proteins were purified with the wild-type BmPSI for EMSA (Figure 2b). EMSA results indicated that five types of mutant proteins lost most of their binding ability compared with the wild-type BmPSI, especially I116G, L127G, and the mut IGGI. 


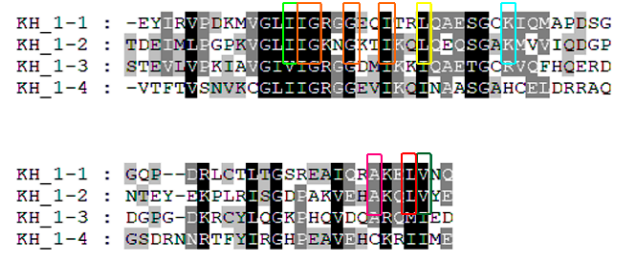

(a)

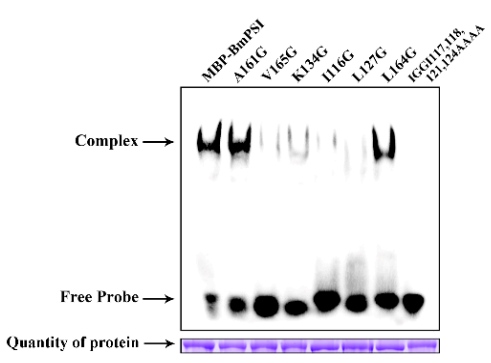

(b)

Figure 2. Important amino acids in the KH_1-1 motif of BmPSI: (a) Comparison among amino acid sequences of the KH_1 motifs in BmPSI. The black color represents that amino acids are completely identical. The gray color represents that partial amino acids are identical. Different colored boxes represent different potential key amino acid sites for the binding. (b) After the concentrations were adjusted uniformly, EMSA was performed on wild-type BmPSI and seven amino acid mutants of BmPSI.

To determine whether these three amino acid mutant proteins transform the secondary structure of BmPSI, circular dichroism (CD) spectroscopy was performed on the wild-type BmPSI and these three mutant proteins (Figure 3a). The results suggest that the three mutant proteins and the wild-type BmPSI have similar patterns. There are two minima at 208 and $222 \mathrm{~nm}$ in the result, which is considered to be the norm for $\alpha$-helices [16]. This suggests that these amino acid mutations did not influence the interaction process by altering the secondary structure of BmPSI. To verify that these three amino acid mutants did lose their ability to bind CE1, isothermal titration calorimetry (ITC) was performed (Figure 3b) $[17,18]$. Firstly, the wild-type BmPSI was analyzed to explore the appropriate conditions for the experiment. Then, the detailed energetic profile of the interaction between BmPSI and CE1 was obtained (Table 1). The average K value of $1.74 \times 10^{7}(n=1.02)$ of the CE1/BmPSI system indicates that there is a strong interaction between BmPSI and CE1.
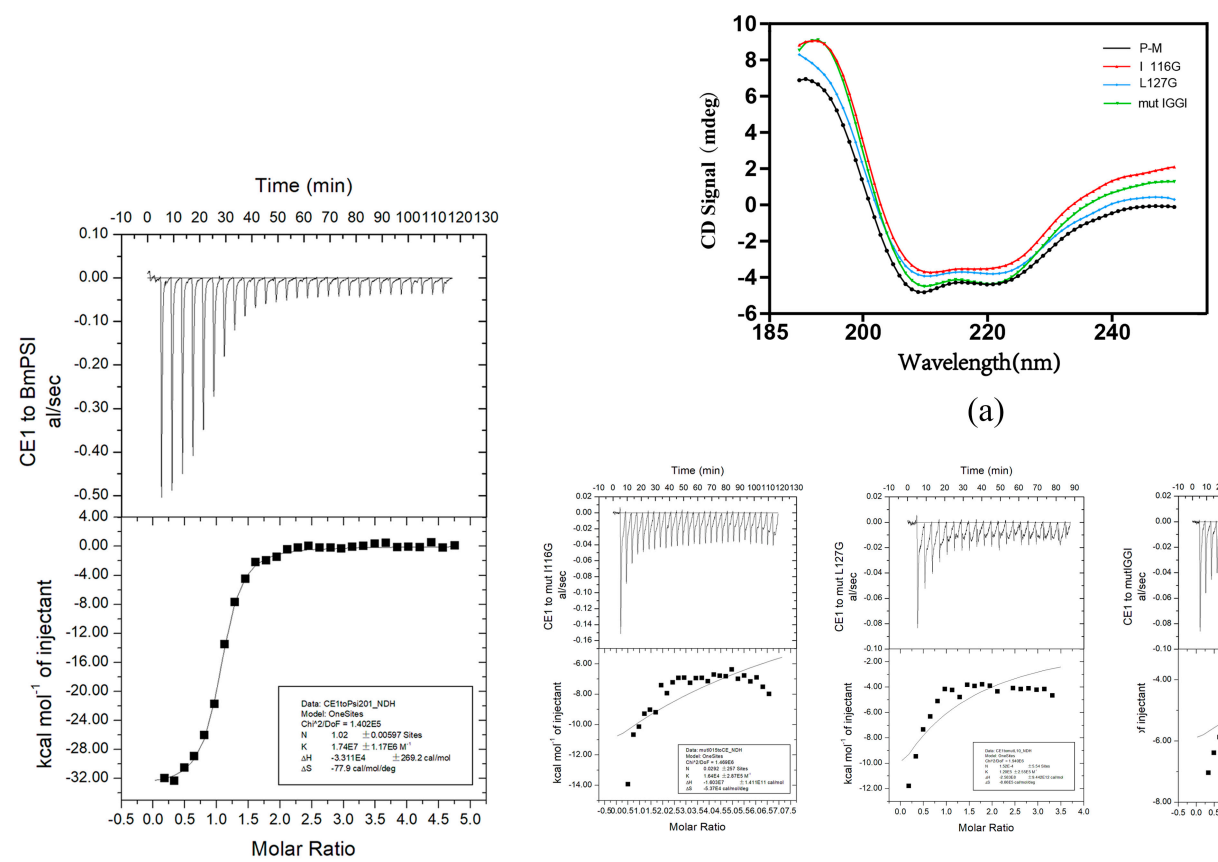

(a)
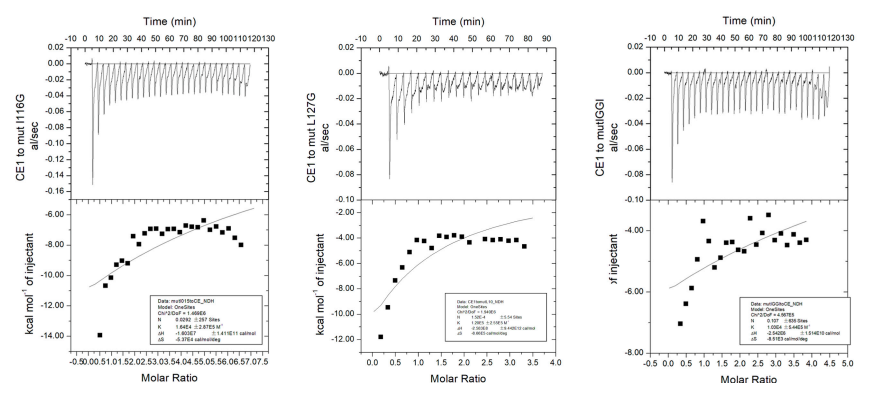

(b)

Figure 3. Two amino acids in the KH_1-1 motif of BmPSI play important roles in the binding with CE1: (a) The secondary structures of wild-type BmPSI and three kind of amino acid mutant proteins detected with circular dichroism (CD) spectroscopy. (b) The RNA binding affinity of three kinds of amino acid mutant proteins and wild-type BmPSI were assessed, showing different exports of isothermal titration calorimetry (ITC) under the same conditions. 
Table 1. The energetic profile of the interaction of the BmPSI with CE1 obtained from ITC.

\begin{tabular}{ccccc}
\hline Molecule & $\boldsymbol{\Delta H}(\mathrm{cal} / \mathbf{m o l})$ & $\boldsymbol{\Delta S}(\mathrm{cal} / \mathbf{m o l} / \mathbf{d e g})$ & $n$ & $\mathbf{K}\left(\mathbf{M}^{-\mathbf{1}}\right)$ \\
\hline BmPSI & $-3.3 \times 10^{4} \pm 269.2$ & -77.9 & 1.02 & $1.74 \times 10^{7}$ \\
\hline
\end{tabular}

The three mutant proteins were analyzed individually with the same conditions grouped from the experiment of BmPSI and CE1. From the ITC results, we found that the ITC data of these three amino acid mutant proteins could not fit the model properly, indicating that these mutant proteins did lose their ability to interact with CE1.

\subsection{The Combination of PSI and CE1 is Conserved in Spodoptera Litura}

Among the three kinds of amino acid mutants, the two which were not IGGI were found close to the Ile-Gly-X2-Gly-X2-Ile structure, which was conserved in the KH_1 motifs. Hence, we considered that the two amino acids near the Ile-Gly-X2-Gly-X2-Ile structure strongly influenced the combination of PSI and RNA in not only B. mori but also other species. A phylogenic tree was performed with these sequences of homolog PSI in ten species (Figure 4a). It was shown that BmPSI, SIPSI, and Helicoverpa armigera PSI (HaPSI) were clustered into the same clade. From the conservative analysis of KH_1 motifs in these ten species, it was found that the KH_1-1 motif of SIPSI was similar to the KH_1-1 of BmPSI (Figure $4 \mathrm{~b}$ ). SIPSI was selected as the experimental subject to verify the importance of key amino acids for the combination of PSI and CE1. It was confirmed that the CE1 exists conservatively in the female-specific splicing of Slds $x$ in S. litura. Full length coding sequence (CDS) of SIPSI was cloned and the SIPSI was purified with Ni-NTA and gel filtration chromatography. Cold competition EMSA and SIPSI concentration gradient EMSA were used to show that the combination of PSI and CE1 was also conserved in S. litura (Figure 4c).

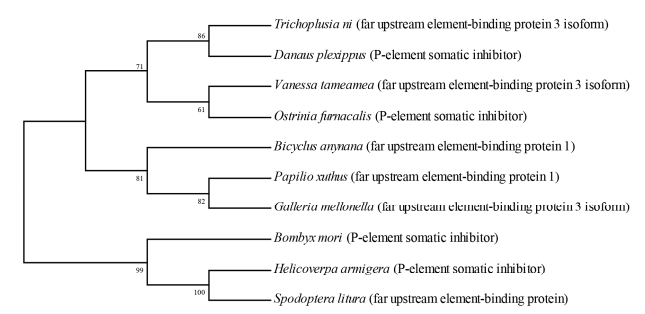

(a)

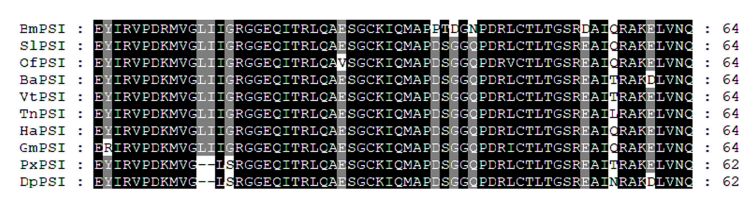

(b)

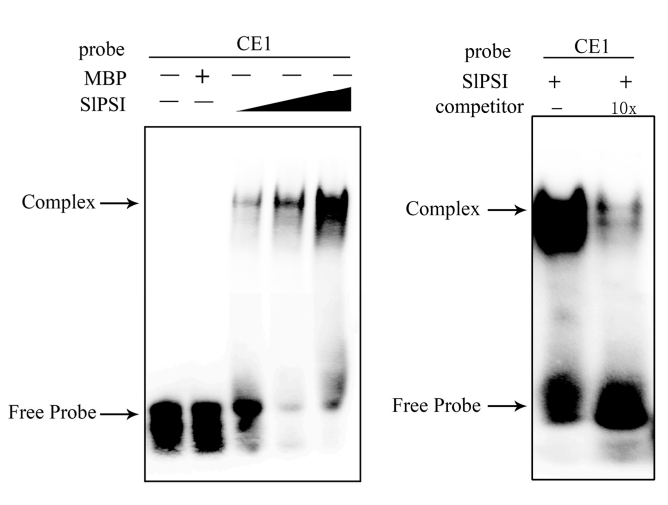

(c)

Figure 4. The combination of PSI and CE1 is conserved in Spodoptera litura: (a) A phylogenic tree based on the PSI of 10 species. (b) Conservation analysis of KH_1 motifs in these 10 species. The black color represents that amino acids are completely identical. The gray color represents that partial amino acids are identical. (c) SIPSI concentration gradient EMSA and cold competition EMSA.

\subsection{Identifying a Key Amino Acid for the Combination of SIPSI and CE1}

Three types of amino acid mutations (L116I, L127G, and IGGI) were added into SIPSI. The mutant proteins were purified and the concentrations of mutant proteins were adjusted consistently for EMSA (Figure 5a). The results showed that mut IGGI can weakly bind to CE1, and that I116G lost most of its ability to interact with CE1 compared to SIPSI. The L127G also lost a lot of its binding capacity but this was not as conspicuous as with I116G. In order to eliminate the effect of nonspecific binding, the SIPSI mutL127G protein was used in a cold competition EMSA experiment (Figure 5b). When the concentration of the competitive probe was aggrandized, the signal intensity of the complex 
decreased sharply. This result showed that the interaction between CE1 and SIPSI mutL127G is specific. To summarize, we found two key amino acids in the KH-1-1 motif of SIPSI. One was the conservative key structure Ile-Gly-X2-Gly-X2-Ile of the $\mathrm{KH}_{-} 1$ motif that has been reported previously [15] and the other was the amino acid I116, which is close to Ile-Gly-X2-Gly-X2-Ile. This is a new discovery of the key amino acid in proteins containing the $\mathrm{KH} \_1$ motif for binding its target RNA.

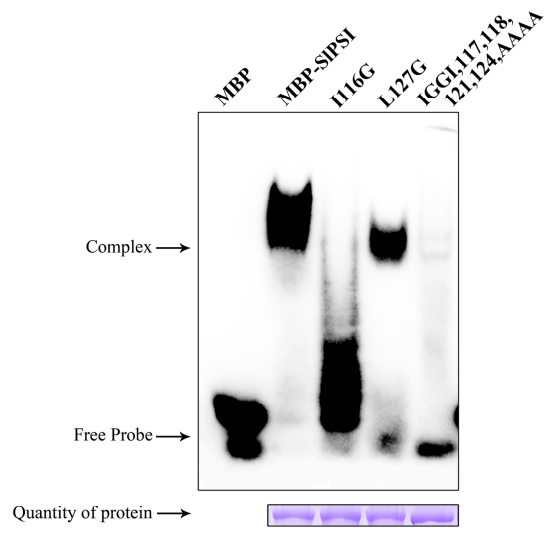

(a)

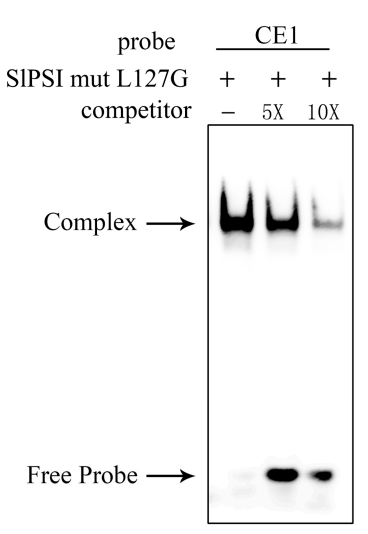

(b)

Figure 5. EMSA of wild-type SIPSI and three kinds of potentially key amino acid mutant SIPSI proteins. (a) MBP-BmPSI was set as the positive control. The signal intensity of the complex stood for the abilities of different proteins to interact with the CE1 probe. (b) Cold competition EMSA experiment between CE1 and SIPSI mutL127G.

\section{Discussion}

BmPSI contains four KH_1 motifs and it is considered to be an informative model for exploring how these $\mathrm{KH}$ motifs contribute to RNA binding and recognition in these proteins with multiple $\mathrm{KH}$ motifs. The EMSA of BmPSI and four truncate BmPSI proteins showed that the four KH_1 motifs participate in the interaction between BmPSI and CE1, in which the KH_1-1 and KH_1-2 play more important roles in the combination process. Through sequence alignment, EMSA, CD spectroscopy, and ITC, we found three potentially key amino acids in the KH_1-1 motif of BmPSI. After verifying the interaction of SIPSI and CE1, these three kinds of amino acid mutations were cloned into SIPSI. Except for the L127G mutation, we found that the I116G and IGGI mutations lost most of their binding ability compared with the wild-type SIPSI.

Drosophila PSI requires multiple tandem KH domains for specific and high-affinity recognition of substrate RNA [11]; however, the KH_1-1 and KH_1-2 motifs of BmPSI play more important roles in the interaction of BmPSI and Bmdsx pre-mRNA compared with the other two KH_1 motifs. Differences in the binding ability of the L127 key site in BmPSI and SIPSI suggest that there are a lot of differences between these two relatively close lepidopterans. The binding affinity of mutant I116G sharply decreased in BmPSI and SIPSI, indicating that this is a key site in the KH_1 motif for the binding process. Furthermore, I116G is stably conserved in Lepidoptera, which indicates it is a key amino acid for binding affinity in proteins containing KH_1 motifs, just as EMSA confirmed it was. I116 is isoleucine, which is tightly close to the Ile-Gly-X2-Gly-X2-Ile structure. These discoveries provide further insight into how $\mathrm{KH} \_1$ motifs contribute to the combining of a protein and its target RNA sequence.

The isoforms of sex-specific alternative splicing of Bmdsx are translated into sex-specific BmDSX proteins which lead to differences in the expression of sex-specific genes, and, thus, sexual differentiation $[19,20]$. BmPSI is the most important part of the exonic splicing silencer complex for binding Bmdsx pre-mRNA with CE1 to promote male-specific splicing of $B m d s x$ [21,22]. This is the only mechanism known as being closely upstream of $B m d s x$ in the sex determination cascade of 
B. mori. The researchers could not generate a gender reversal strain through genetic modification of $B m d s x$ or the knockout of Bmpsi. Two possible reasons for this are that Bmdsx leads the expressions of many sex-specific genes and Bmpsi participates in not only sex determination but also other key pathways in B. mori. Therefore, a slight change in Bmdsx or Bmpsi would cause disorder in transgene stains. Thus, the key amino acid sites of the interaction between BmPSI and CE1 could be used to establish transgenic strains, in which the transgenic BmPSI loses its capacity to bind to CE1 but retains its other functions.

From an evolutionary perspective, the four conserved KH_1 motifs in SIPSI and CE1 in female specific splicing of Slds $x$ provide strong evidence of conserved structures for essential components of the sex determination process in Lepidoptera [23]. Furthermore, the conserved interaction of SIPSI and CE1 indicates functional conservation of essential components in the sex determination process in Lepidoptera. In addition, the discovery of this key site for the binding in both BmPSI and SIPSI not only contributes a lot to future research on sex determination of lepidopterans but also provides a theoretical basis for gender reversal in Bombyx mori $[24,25]$.

\section{Materials and Methods}

\subsection{Construction of Recombinant Expression Vectors}

The $\mathrm{pET} \mathrm{His}_{6}$ MBP TEV LIC cloning vector is an LIC N-terminal fusion vector for E. coli expression which contains a maltose binding protein. The wild type Bmpsi sequence was cloned into this vector behind a (His) 6 affinity tag by LIC cloning. Four different KH_1 region truncations of Bmpsi were made with overlapping PCR. Seven point mutations in the KH_1-1 motif of Bmpsi were added with site-directed mutagenesis into the recombinant vector containing Bmpsi (Table 2).

Table 2. Primer sequences of modifications on Bmpsi.

\begin{tabular}{|c|c|}
\hline Name & Primers \\
\hline Bmpsi & $\begin{array}{r}5^{\prime} \text {-cgcggatccatgagtgattattcttctat-3', } \\
5^{\prime} \text {-ccgctcgagtcactgctggtggtcggagc- } 3^{\prime}\end{array}$ \\
\hline$\Delta \mathrm{KH} \_1-1$ & $\begin{array}{c}5^{\prime} \text {-cgatcagagcatcaatattgtgaatcatcgaggaa-3' } \\
5^{\prime} \text {-ttcctcgatgattcacaatattgatgctctgatcg-3' }\end{array}$ \\
\hline$\Delta \mathrm{KH} \_1-2$ & $\begin{array}{l}5^{\prime} \text {-ccaggccetaatgcaatgctcctcgccaacaagga-3' } \\
5^{\prime} \text {-tccttgttggcgaggagcattgcattagggcctgg-3' }\end{array}$ \\
\hline$\Delta \mathrm{KH} \_1-3$ & $\begin{array}{l}5^{\prime} \text {-aacggactcgccaccactcttatatctagtgtcaa-3' } \\
5^{\prime} \text {-ttgacactagatataagagtggtggcgagtccgtt-3' }\end{array}$ \\
\hline$\Delta \mathrm{KH} \_1-4$ & $\begin{array}{l}5^{\prime} \text {-gaccgaccagagatgcgcaaagttggtgggcetgt- } 3^{\prime} \\
5^{\prime} \text {-acaggcccaccaactttgcgcatctctggtcggtc }-3^{\prime}\end{array}$ \\
\hline I116G & $\begin{array}{l}5^{\prime} \text {-gttggactaggaattggacgtggt- } 3^{\prime} \\
5^{\prime} \text {-accacgtccaattcctagtccaac- } 3^{\prime}\end{array}$ \\
\hline L127G & $\begin{array}{l}5^{\prime} \text {-atcaccagagggcaagcagaatcc- } 3^{\prime} \\
5^{\prime} \text {-ggattctgcttgccctctggtgat- } 3^{\prime}\end{array}$ \\
\hline K134G, & $\begin{array}{l}5^{\prime} \text {-tccggttgcgggatacaaatggca- } 3^{\prime} \\
5^{\prime} \text {-tgccatttgtatcccgcaaccgga- } 3^{\prime}\end{array}$ \\
\hline A161G & $\begin{array}{l}5^{\prime} \text {-atacagagaggtaaagaattagtg- } 3^{\prime} \\
5^{\prime} \text {-cactaattctttacctctctgtat- } 3^{\prime}\end{array}$ \\
\hline L164G & $\begin{array}{c}5^{\prime} \text {-gctaaagaaggagtgaaccaaatt-3' } \\
5^{\prime} \text {-aatttggttcactccttctttagc-3' }\end{array}$ \\
\hline V165G & $\begin{array}{l}5^{\prime} \text {-aaagaattagggaaccaaattgtg- } 3^{\prime} \\
5^{\prime} \text {-cacaatttggttccctaattcttt- } 3^{\prime}\end{array}$ \\
\hline IGGI 117, 118, 121, 124 AAAA & $\begin{array}{c}5^{\prime} \text {-ggactaatagctgcacgtggtgga-3' } \\
5^{\prime} \text {-tccaccacgtgcagctattagtcc-3', } \\
5^{\prime} \text {-cacgtggtgcagaacaagccaccagactgca-3' } \\
5^{\prime} \text {-tgcagtctggtggcttgttctgcaccacgtg-3', } \\
5^{\prime} \text { - gcagaacaagccaccagactgcaa-3' } \\
5^{\prime} \text {-ttgcagtctggtggcttgttctgc-3' }\end{array}$ \\
\hline
\end{tabular}


The sequence of Slpsi was downloaded from NCBI and was predicted to be the S. litura far upstream element-binding protein (LOC 111350908). According to the sequence information on NCBI, we designed a pair of primers to clone the fragment of Slpsi into the pET His ${ }_{6}$ MBP TEV LIC cloning vector. Three kinds of point mutation in the KH_1-1 motif of Slpsi were added with site-directed mutagenesis into the recombinant vector (Table 3).

Table 3. Primer sequences of modifications on Slpsi

\begin{tabular}{|c|c|}
\hline Name & Primers \\
\hline Slpsi & $\begin{array}{c}5^{\prime} \text { - cgggatccatgagtgattattcttctatggc- } 3^{\prime} \\
5^{\prime} \text {-ggaattcctattgatgatggtcggg- } 3^{\prime}\end{array}$ \\
\hline I116G & $\begin{array}{l}5^{\prime} \text {-atggttggactcggaattgggcgcggc-3' } \\
5^{\prime} \text {-gccgcgcccaattccgagtccaaccat-3' }\end{array}$ \\
\hline L127G & $\begin{array}{c}5^{\prime} \text {-atcacacgcgggcaggcggagtca-3' } \\
5^{\prime} \text {-tgactccgcctgcccgcgtgtgat-3' }\end{array}$ \\
\hline IGGI $117,118,121,124$ AAAA & $\begin{array}{l}5^{\prime} \text {-ggactcatagctgggcgcggcggtgag- } 3^{\prime} \\
5^{\prime} \text {-ctcaccgccgcgcccagctatgagtcc-3', } \\
5^{\prime} \text {-ggactcatagctgcacgcggcggtgag-3' } \\
5^{\prime} \text {-ctcaccgccgcgtgcagctatgagtcc-3', } \\
5^{\prime} \text {-gcacgcggcgcagagcaggccacacgc-3' } \\
5^{\prime} \text {-gcgtgtggcctgctctgcgccgcgtgc- } 3^{\prime}\end{array}$ \\
\hline
\end{tabular}

\subsection{Preparation of Single Stranded RNA}

Two kinds of CE1 single-stranded RNA were prepared for EMSA and ITC. One kind of CE1 RNA was labeled with Biotin on its $5^{\prime}$ end and $3^{\prime}$ end. The sequence of the single-stranded RNA was the same as the sequence of CE1 in the female-specific splicing of $B m d s x$ ( $5^{\prime}$-uuaauaauauaaguggugua- $\left.3^{\prime}\right)$. The RNA probe was compounded by the Beijing Genomics Institute and purified with ion exchange HPLC. The RNA was dissolved in a buffer $(20 \mathrm{mM}$ Tris, $20 \mathrm{mM} \mathrm{NaCl})$, which was used to purify the different kinds of protein.

\subsection{The Overexpression and Purification of Different Kinds of Proteins}

Transetta (DE3) Chemically Competent Cell was used to overexpress the wild-type BmPSI, the wild-type SIPSI, the four KH_1 region truncation proteins of BmPSI, the seven amino acid mutants of BmPSI proteins, and the three amino acid mutants of SIPSI proteins with the recombinant vectors discussed above. We overexpressed the maltose-binding protein with the original expression vector as a negative control. The purification of every kind of protein needed $2 \mathrm{~L}$ cells in LB media at $37^{\circ} \mathrm{C}$ with an absorbance at $600 \mathrm{~nm}$ of 0.5 . Then, we added Isopropyl $\beta$-D-Thiogalactoside (IPTG)to a final concentration of $1 \mathrm{mM}$ and cultured the cells at $37^{\circ} \mathrm{C}$ for another $4 \mathrm{~h}$. After cell culturing, the cells were gathered and crushed with multi-gelation three times and ultra-sonicated (40\%, $30 \mathrm{~min})$. The supernatant was separated using centrifugation $(15,000 \times g, 10 \mathrm{~min})$. A series of buffers with different concentrations of imidazole were used to wash the HiTrap Chelating where the supernatant was loaded. The buffer containing the target protein was passed through gel filtration chromatography, which made it purer. Finally, we retrieved the pure target protein with the (His) 6 -MBP-TEV component.

\subsection{Bioinformatics Analysis}

The sequences of the four KH_1 motifs in BmPSI were compared to find out the potential key amino acids which are conservative in KH_1-1and KH_1-2 but not KH_1-3 and KH_1-4 [26-28]. The PSI sequences of ten species were downloaded from NCBI to construct a phylogenic tree $[29,30]$ and the sequences of the four $\mathrm{KH} \_1$ motifs from BmPSI were compared by multiple sequence alignment. 


\subsection{Electrophoretic Mobility Shift Assay}

Electrophoretic mobility shift assay can be used to determine binding affinity, specificity, and stoichiometry of the RNA/protein interaction [31-33]. In this article, we used EMSA experiments in different ways which were designed to accommodate two different experimental objectives. A normal EMSA was used to verify the interaction between SIPSI and CE1 with cold competition EMSA and SIPSI concentration gradient EMSA. The other kind of EMSA was designed to compare the binding affinity with RNA of different proteins under the same conditions. At first, the concentrations of proteins in every group were adjusted consistently with BCA assay and SDS-PAGE. Then, the proteins were experimented on under exactly the same conditions. As a result, the signal intensity of the complex stood for the abilities of different proteins to interact with the RNA probe. One group of proteins included MBP, MBP-BmPSI, and four KH_1 region truncate BmPSI proteins, which were set to compare the binding abilities of different KH_1 motifs. Another group of proteins included MBP-BmPSI and the seven amino acid mutant BmPSI proteins, which were set to show the importance these key amino acids had in the interaction between BmPSI and CE1. The third group of proteins included MBP-SIPSI and the three amino acid mutant SIPSI proteins, which were set to detect the conservative property of the key amino acids we found in BMPSI for the combination of PSI and CE1 in other species.

\subsection{Wavelength Scans}

Circular dichroism spectroscopy is a technique which is widely used to detect secondary structure composition. The wild-type BmPSI protein and the three kinds of amino acid mutations of BmPSI, which were important to the interaction between BmPSI and CE1, were experimented on for these secondary structures with $C D$ wavelength scans. Before the $C D$ wavelength test, the concentrations of different proteins were adjusted consistently with a BCA protein assay kit and SDS-PAGE.

\subsection{Isothermal Titration Calorimetry}

Isothermal titration calorimetry is always used to estimate binding ability through the binding enthalpy $(\Delta \mathrm{H})$ [34]. An ITC isotherm of the CE1/BmPSI system was performed at $25^{\circ} \mathrm{C}$ with an cell volume of $1.4 \mathrm{~mL}$ using a stirring rate of $307 \mathrm{rpm}$. The CE1 probe was injected $2 \mu \mathrm{L}$ at a time into a solution of BmPSI at $210 \mathrm{~s}$ to allow complete equilibration of the CE1/BmPSI system between each injection. The most appropriate experimental conditions were grouped with the experiment between wild-type BmPSI and the CE1 probe. Then, the other amino acid mutants with the same concentration were experimented on under the same conditions. The differences in binding ability among these four proteins were determined from the curves constructed during these ITC experiments.

Author Contributions: Data curation, Y.W.; formal analysis, Y.W.; funding acquisition, X.-F.Z.; investigation, X.-F.Z.; methodology, Y.W. and X.-F.Z.; software, Y.W.; validation, Y.W.; writing-original draft, Y.W.; writing-review and editing, Q.Z., Q.-X.W., K.-X.W., and X.-F.Z.

Funding: This research was funded by the State Key Program of National Natural Science of China (grant no. 31530071) and the National Natural Science Foundation of China (grant no. 31272502).

Acknowledgments: We thank Lin Liu (South China Normal University) for providing the sequence of the female-specific splicing of Sldsx.

Conflicts of Interest: The authors declare no conflict of interest.

\section{Abbreviations}

$\begin{array}{ll}\text { dsx } & \text { Doublesex } \\ \text { PSI } & \text { P-element somatic inhibitor } \\ \text { CD } & \text { Circular dichroism } \\ \text { ITC } & \text { Isothermal titration calorimetry } \\ \text { EMSA } & \text { Electrophoretic mobility shift assay }\end{array}$




\section{References}

1. Ohbayashi, F.; Suzuki, M.G.; Mita, K.; Okano, K.; Shimada, T. A homologue of the Drosophila doublesex gene is transcribed into sex-specific mRNA isoforms in the silkworm, Bombyx mori. Comp. Biochem. Phys. B 2001, 128, 145-158. [CrossRef]

2. Suzuki, M.G.; Funaguma, S.; Kanda, T.; Tamura, T.; Shimada, T. Role of the male BmDSX protein in the sexual differentiation of Bombyx mori. Evol. Dev. 2005, 7, 58-68. [CrossRef]

3. Funaguma, S.; Suzuki, M.G.; Tamura, T.; Shimada, T. The Bmdsx transgene including trimmed introns is sex-specifically spliced in tissues of the silkworm, Bombyx mori. J. Insect Sci. 2005, 5, 17-22. [CrossRef]

4. Suzuki, M.G.; Ohbayashi, F.; Mita, K.; Shimada, T. The mechanism of sex-specific splicing at the doublesex gene is different between Drosophila melanogaster and Bombyx mori. Insect Biochem. Mol Biol. 2001, 31, 1201-1211. [CrossRef]

5. Suzuki, M.G.; Imanishi, S.; Dohmae, N.; Nishimura, T.; Shimada, T.; Matsumoto, S. Establishment of a novel in vivo sex-specific splicing assay system to identify a trans-acting factor that negatively regulates splicing of Bombyx mori dsx female exons. J. Mol. Cell Biol. 2008, 28, 333-343. [CrossRef]

6. Xu, J.; Chen, S.; Zeng, B.; James, A.A.; Tan, A.; Huang, Y. Bombyx mori P-element somatic inhibitor (BmPSI) is a key auxiliary factor for silkworm male sex determination. PLoS Genet. 2017, 13, e1006576. [CrossRef] [PubMed]

7. Adinolfi, S.; Bagni, C.; Castiglione Morelli, M.A.; Fraternali, F.; Musco, G.; Pastore, A. Novel RNA-binding motif: The KH module. Biopolymers 1999, 51, 153-164. [CrossRef]

8. Fraternali, F.; Amodeo, P.; Musco, G.; Nilges, M.; Pastore, A. Exploring protein interiors: The role of a buried histidine in the KH module fold. Proteins 1999, 34, 484-496. [CrossRef]

9. Hall, M.P.; Huang, S.; Black, D.L. Differentiation-induced colocalization of the KH-type splicing regulatory protein with polypyrimidine tract binding protein and the c-src pre-mRNA. Mol. Biol. Cell 2004, 15, 774-786. [CrossRef] [PubMed]

10. Art, J.; Besche, V.; Bros, M.; Li, H.; Handler, N.; Bauer, F.; Erker, T.; Behnke, F.; Monch, B.; Forstermann, U.; et al. Identification of the $\mathrm{KH}$ type splicing regulatory protein (KSRP) as a new important mediator of the anti-inflammatory effects of resveratrol. N.-S. Arch. Pharmacol. 2012, 385, 6.

11. Chmiel, N.H.; Rio, D.C.; Doudna, J.A. Distinct contributions of KH domains to substrate binding affinity of Drosophila P-element somatic inhibitor protein. RNA 2006, 12, 283-291. [CrossRef]

12. Guo, L.; Zaysteva, O.; Nie, Z.; Mitchell, N.C.; Amanda Lee, J.E.; Ware, T.; Parsons, L.; Luwor, R.; Poortinga, G.; Hannan, R.D.; et al. Defining the essential function of FBP/KSRP proteins: Drosophila Psi interacts with the mediator complex to modulate MYC transcription and tissue growth. Nucleic Acids Res. 2016, 44, 7646-7658. [CrossRef]

13. Labourier, E.; Blanchette, M.; Feiger, J.W.; Adams, M.D.; Rio, D.C. The KH-type RNA-binding protein PSI is required for Drosophila viability, male fertility, and cellular mRNA processing. Gene Dev. 2002, 16, 72-84. [CrossRef]

14. Makeyev, A.V.; Eastmond, D.L.; Liebhaber, S.A. Targeting a KH-domain protein with RNA decoys. RNA 2002, 8, 1160-1173. [CrossRef]

15. Xu, H.X.; Xu, Y.J.; Liang, X.J.; Zeng, W.X. The biological function of heterogeneous nuclear ribonucleoprotein $\mathrm{K}$ (hnRNP K) and its roles in spermatogenisis. J. Agric. Biotechnol. 2015, 23, 661-670.

16. Micsonai, A.; Wien, F.; Kernya, L.; Lee, Y.H.; Goto, Y.; Refregiers, M.; Kardos, J. Accurate secondary structure prediction and fold recognition for circular dichroism spectroscopy. Proc. Natl. Acad. Sci. USA 2015, 112, E3095-E3103. [CrossRef]

17. Arroyo-Maya, I.J.; McClements, D.J. Application of ITC in foods: A powerful tool for understanding the gastrointestinal fate of lipophilic compounds. BBA-Gen. Subjects 2016, 1860, 1026-1035. [CrossRef]

18. Johnson, R.A.; Manley, O.M.; Spuches, A.M.; Grossoehme, N.E. Dissecting ITC data of metal ions binding to ligands and proteins. BBA-Gen. Subjects 2016, 1860, 892-901. [CrossRef]

19. Kawaoka, S.; Kadota, K.; Arai, Y.; Suzuki, Y.; Fujii, T.; Abe, H.; Yasukochi, Y.; Mita, K.; Sugano, S.; Shimizu, K.; et al. The silkworm $\mathrm{W}$ chromosome is a source of female-enriched piRNAs. RNA 2011, 17, 2144-2151. [CrossRef] 
20. Hara, K.; Fujii, T.; Suzuki, Y.; Sugano, S.; Shimada, T.; Katsuma, S.; Kawaoka, S. Altered expression of testis-specific genes, piRNAs, and transposons in the silkworm ovary masculinized by a W chromosome mutation. BMC Genomics 2012, 13, 119-128. [CrossRef]

21. Suzuki, M.G.; Imanishi, S.; Dohmae, N.; Asanuma, M.; Matsumoto, S. Identification of a male-specific RNA binding protein that regulates sex-specific splicing of Bmdsx by increasing RNA binding activity of BmPSI. Mol. Cell Biol. 2010, 30, 5776-5786. [CrossRef]

22. Kiuchi, T.; Koga, H.; Kawamoto, M.; Shoji, K.; Sakai, H.; Arai, Y.; Ishihara, G.; Kawaoka, S.; Sugano, S.; Shimada, T.; et al. A single female-specific piRNA is the primary determiner of sex in the silkworm. Nature 2014, 509, 633-636. [CrossRef]

23. Du, Q.; Wen, L.; Zheng, S.C.; Bi, H.L.; Huang, Y.P.; Feng, Q.L.; Liu, L. Identification and functional characterization of doublesex gene in the testis of Spodoptera litura. Insect Sci. 2018. [CrossRef]

24. Nagashim, E.; Hujieda, T.; Moriizum, S. On expression of several characters affected by difference of sexes in silkworm. Jpn. J. Genet. 1967, 42, 427.

25. Shabdini, A.; Seidavi, A.R.; Gharahveysi, S.; Mirhosseini, S.Z. Effects of sex, generation and genetic group on economic traits in three Iranian commercial silkworm pure lines. J. Anim. Vet. Adv. 2011, 10, 76-80. [CrossRef]

26. Amorim, A.R.; Neves, L.A.; Valencio, C.R.; Roberto, G.F.; Zafalon, G.F.D. An approach for COFFEE objective function to global DNA multiple sequence alignment. Comput. Biol. Chem. 2018, 75, 39-44. [CrossRef]

27. Nute, M.; Saleh, E.; Warnow, T. Evaluating statistical multiple sequence alignment in comparison to other alignment methods on protein data sets. Syst. Biol. 2018, 68, 396-411. [CrossRef] [PubMed]

28. Dong, D.; Su, W.; Shi, W.; Zou, Q.; Peng, S. VCSRA: A fast and accurate multiple sequence alignment algorithm with a high degree of parallelism. J. Genet. Genomics 2018, 45, 407-410. [CrossRef]

29. Mirkov, T.E.; Wahlstrom, J.M.; Hagiwara, K.; Finardi-Filho, F.; Kjemtrup, S.; Chrispeels, M.J. Evolutionary relationships among proteins in the phytohemagglutinin-arcelin-alpha-amylase inhibitor family of the common bean and its relatives. Plant. Mol. Biol. 1994, 26, 1103-1113. [CrossRef]

30. Lai, X.; Zhou, Y.; Xia, X.; Zhang, Q. Performance analysis of evolutionary algorithms for steiner tree problems. Evol. Comput. 2017, 25, 707-723. [CrossRef]

31. Hellman, L.M.; Fried, M.G. Electrophoretic mobility shift assay (EMSA) for detecting protein-nucleic acid interactions. Nat. Protoc. 2007, 2, 1849-1861. [CrossRef] [PubMed]

32. Jing, D.; Agnew, J.; Patton, W.F.; Hendrickson, J.; Beechem, J.M. A sensitive two-color electrophoretic mobility shift assay for detecting both nucleic acids and protein in gels. Proteomics 2003, 3, 1172-1180. [CrossRef] [PubMed]

33. Fillebeen, C.; Wilkinson, N.; Pantopoulos, K. Electrophoretic mobility shift assay (EMSA) for the study of RNA-protein interactions: The IRE/IRP example. J. Vis. Exp. 2014, 94, e52230. [CrossRef] [PubMed]

34. Jensen, L.B.; Mortensen, K.; Pavan, G.M.; Kasimova, M.R.; Jensen, D.K.; Gadzhyeva, V.; Nielsen, H.M.; Foged, C. Molecular characterization of the interaction between siRNA and PAMAM G7 dendrimers by SAXS, ITC, and molecular dynamics simulations. Biomacromolecules 2010, 11, 3571-3577. [CrossRef] [PubMed]

(C) 2019 by the authors. Licensee MDPI, Basel, Switzerland. This article is an open access article distributed under the terms and conditions of the Creative Commons Attribution (CC BY) license (http://creativecommons.org/licenses/by/4.0/). 